\title{
H3Africa: current perspectives
}

This article was published in the following Dove Press journal:

Pharmacogenomics and Personalized Medicine

\author{
Nicola Mulder' \\ Alash'le Abimiku² \\ Sally N Adebamowo ${ }^{3}$ \\ Jantina de Vries ${ }^{4}$ \\ Alice Matimba ${ }^{5}$ \\ Paul Olowoyo ${ }^{6}$ \\ Michele Ramsay ${ }^{7}$ \\ Michelle Skelton' \\ Dan J Stein ${ }^{8,9}$
}

On behalf of the members of the H3Africa Consortium

'Computational Biology Division, Department of Integrative Biomedical Sciences, Institute of Infectious Disease and Molecular Medicine, University of Cape Town, Cape Town, South Africa; ${ }^{2}$ nternational Research Center of Excellence, Institute of Human Virology, Abuja, Nigeria; ${ }^{3}$ Department of Epidemiology and Public Health, University of Maryland School of Medicine, Baltimore, MD, USA; ${ }^{4}$ Department of Medicine, University of Cape Town, Cape Town, South Africa;

${ }^{5}$ Advanced Courses and Scientific Conferences, Wellcome Genome Campus, Hinxton, UK; ${ }^{6}$ Federal Teaching Hospital, College of Medicine and Health Sciences, Afe Babalola University, Ado-Ekiti, Nigeria; ${ }^{7}$ Sydney Brenner Institute for Molecular Bioscience, Division of Human Genetics, Faculty of Health Sciences, University of the Witwatersrand, Johannesburg, ${ }^{8}$ Department of Psychiatry and Mental Health, University of Cape Town, ${ }^{9}$ MRC Unit on Risk and Resilience in Mental Disorders, Cape Town, South Africa

Correspondence: Nicola Mulder

Computational Biology Division,

Department of Integrative Biomedical

Sciences, Institute of Infectious Disease and

Molecular Medicine, University of Cape

Town, Anzio Road, Observatory, Cape

Town 7925, South Africa

Tel +272I 4066058

Email nicola.mulder@uct.ac.za
Abstract: Precision medicine is being enabled in high-income countries by the growing availability of health data, increasing knowledge of the genetic determinants of disease and variation in response to treatment (pharmacogenomics), and the decreasing costs of data generation, which promote routine application of genomic technologies in the health sector. However, there is uncertainty about the feasibility of applying precision medicine approaches in low- and middle-income countries, due to the lack of population-specific knowledge, skills, and resources. The Human Heredity and Health in Africa (H3Africa) initiative was established to drive new research into the genetic and environmental basis for human diseases of relevance to Africans as well as to build capacity for genomic research on the continent. Precision medicine requires this capacity, in addition to reference data on local populations, and skills to analyze and interpret genomic data from the bedside. The H3Africa consortium is collectively processing samples and data for over 70,000 participants across the continent, accompanied in most cases by rich clinical information on a variety of non-communicable and infectious diseases. These projects are increasingly providing novel insights into the genetic basis of diseases in indigenous populations, insights that have the potential to drive the development of new diagnostics and treatments. The consortium has also invested significant resources into establishing high-quality biorepositories in Africa, a bioinformatic network, and a strong training program that has developed skills in genomic data analysis and interpretation among bioinformaticians, wet-lab researchers, and health-care professionals. Here, we describe the current perspectives of the H3Africa consortium and how it can contribute to making precision medicine in Africa a reality.

Keywords: H3Africa, genomic medicine, precision medicine, training, population genetics, disease

\section{Introduction}

Health care and medical practice currently apply prevention and treatment strategies for the average person or patient. Precision medicine, also referred to as precise personalized patient care, includes approaches in diagnostics, prevention, screening, and treatment that involve individually tailoring health care based on an individual's genes, lifestyle, and environment. ${ }^{1}$ Although the concept is not new, advances in genomic technologies and data sciences are enabling more targeted disease diagnostics and treatment approaches that can inform health and disease management at the individual level. Molecular approaches in precision medicine include genomic sequencing, metagenomics, gene therapy, induced pluripotent-cell therapy, and pharmacogenomics. The implementation of precision medicine requires prior knowledge of actionable variants, among other things, as well as significant resources for the clinician. ${ }^{2}$ In the specific case of genomic medicine, substantial knowledge about reference populations of relevance to the individual being treated and variants known to be associated with 
disease risk or adverse drug reactions, along with associated evidence, is required in order to make informed decisions. Clinicians need access to genetic tests, sequencing/array technologies, data analysis capacity to analyze and interpret the results, and often also decision support tools for translating the data into a potential clinical decision. While health-care professionals in developed countries are increasingly being given access to such facilities and expertise, the relevant skills and infrastructure for these activities are currently lacking on the African continent, and reference data and scientific knowledge on African-specific disease variants are scarce. Additionally, the data used to inform precision medicine are largely skewed toward European populations, where the number of potential candidate variants associated with diseases tends to be lower than in other populations, such as those of African descent. ${ }^{3}$ Petrovski and Goldstein ${ }^{3}$ evaluated the effect of geographic ancestry on interpretation of genomic data, and found that bias toward European populations had a negative effect on identifying candidate variants in other populations. For African populations, which have a disproportionate burden of disease, the high genetic diversity provides greater rationale for using genomics to improve our understanding of the genetic basis for diseases, both communicable and non-communicable, and to use the data to guide personalized medicine.

Some of these obstacles to the implementation of precision medicine in Africa are being addressed by several new large-scale initiatives, such as the Human Heredity and Health in Africa (H3Africa) initiative. ${ }^{4}$ The H3Africa initiative is funded by the National Institutes of Health (NIH) and Wellcome Trust to facilitate innovative research into the genetic and environmental basis for diseases affecting African populations. In addition to funding the scientific research, the initiative is developing research infrastructure, including boosting research capacity at individual institutions and three biorepositories for storing project biospecimens as a shared resource. There is also a strong element of human capacity development across the projects and consortium as a whole. H3Africa is focusing on identification of sequence variants in African populations that may contribute to health and disease using high-throughput sequencing and genotyping techniques. In addition to studies on host genetic factors, microbiome projects bring valuable insights to the interplay between microbes and the host's genetic makeup. To facilitate local analysis of the data generated, the NIH has funded H3ABioNet, a pan-African bioinformatic network to build capacity for genomic research on the continent. ${ }^{5} \mathrm{H} 3 \mathrm{ABio}-$ Net is training bioinformaticians, scientists, and health-care professionals in all aspects of data management, analysis, and interpretation, and developing the infrastructure for large-scale data analysis in Africa.

Discoveries from H3Africa research will be used to develop and improve disease diagnostics, to explore the use of genetic risk-prediction algorithms for complex traits in African populations, and to augment drug discovery and development. However, the major impact of the initiative has already been much broader than just scientific discovery. The consortium has worked together to overcome numerous challenges from ethics consent to biospecimen and data transfer. It has built significant capacity in research and human infrastructure and produced valuable cohorts and data that lay the foundations for the realization of precision medicine. Here, we provide some current perspectives on the H3Africa consortium, challenges it has overcome, and some major achievements.

\section{Preliminary discoveries from genomic research in H3Africa}

$\mathrm{H} 3$ Africa projects are focusing on determining the genetic and environmental factors associated with a diverse spectrum of communicable and non-communicable diseases. The latter include kidney disease, diabetes, and a range of cardiovascular diseases (CVDs). Premature death from non-communicable diseases is on the rise in Africa ${ }^{6}$ and has prompted six H3Africa research teams to join forces to establish a combined African resource to enhance CV health research. ${ }^{7,8}$ The H3Africa Cardiovascular Disease Working Group is harmonizing data and pooling resources to establish the Cardiovascular H3Africa Innovation Resource (CHAIR) that will include over 50,000 participants to study genomic and environmental contributions to CVD in Africa. Participants from 15 African countries will have data relevant to the $\mathrm{CV}$ and metabolic disease spectrum, including sociodemographic, anthropometric, clinical, biochemical (blood and urine biomarkers), and genomic data, in addition to stored biological samples for future assays. CHAIR is ideally placed for meta-analysis of genome-wide association-study data and will provide an opportunity to identify important gene-gene and gene-environment interactions. It will be used extensively for non-communicable disease-related research and has the potential to inform precision medicine approaches for complex traits and diseases, including hypertension, dyslipidemia, diabetes, stroke, and kidney disease. ${ }^{8}$

Preliminary data from the H3Africa Genomic and Environmental Risk Factors for Cardiometabolic Disease in Africans (AWI-Gen), a population cross-sectional study of over 
12,000 adult men and women from six sites in four African countries, have revealed extensive regional variation and sex-specific differences for cardiometabolic disease-related risk factors. ${ }^{9,10}$ The prevalence of hypertension ranged from $15.1 \%$ in the rural Burkina Faso center to $54.1 \%$ in urban Soweto, South Africa, with higher proportions in older participants and men generally being less aware of their hypertensive state $(39.4 \%)$ than women $(53.8 \%) .{ }^{10}$ In three centers, women were more likely to be hypertensive, in one it was the reverse, and in the remaining two there was no difference between the sexes, pointing to important interactions and highlighting the need for regionally tailored intervention and prevention strategies.

The H3Africa Stroke Investigative Research \& Educational Network (SIREN) study has identified novel genes associated with stroke. For example, $A P O L 1, C D K N 2 A / C D K N 2 B$, and $H D A C 9$ polymorphisms have been associated with smallvessel ischemic stroke, and IL6 (rs1800796) and CDKN2A/ $C D K N 2 B$ (rs2383207) have been found to be associated with ischemic stroke in indigenous west African men. ${ }^{11}$ The SIREN project has developed a robust imaging database with the use of ACCESS software, ${ }^{12}$ which is user-friendly and able to facilitate concordant and reproducible classification of all stroke types and ischemic stroke subtypes by multiple investigators. It has multilevel adjudication/quality control of image reports in the diagnosis of stroke and exclusion of stroke mimics.

The H3Africa SAX study, which aims to identify genes responsible for schizophrenia, has not yet reported on genetic findings. However, the study has contributed a number of papers on schizophrenia symptomatology, ${ }^{13}$ community engagement, ${ }^{14}$ and ethical issues. For example, given previous work on the relationship between schizophrenia and cultural beliefs, we undertook a study of the content of delusions in a subsample of participants. In this subsample, the majority of research participants $(72.5 \%)$ believed that others had bewitched them in order to bring about their mental illness, because they were in some way jealous of the participant. This explanation aligns well with the understanding of jealousy-induced witchcraft in southern African communities, and highlights the important role that culture plays in the content of delusions. ${ }^{13}$

Communicable diseases are also a focus of some of the H3Africa projects, with studies on the epigenetics of trypanosomiasis, HIV, and surveillance of microbial threats, among others. Funding for the surveillance project that is led from Nigeria helped to develop skills and infrastructure for pathogen research in this region, and this team was instrumental in responding to the recent Ebola outbreak.
The 2013-2016 Ebola virus disease epidemic in west Africa was unprecedented, claiming 11,323 lives to date. ${ }^{15}$ While the situation was exacerbated by the ill-preparedness of the global community, it highlighted the significant contribution and proficiency of African scientists nested within the epidemic and the importance of committed international collaboration. The combined efforts of domestic west African research expertise and international partners supported the formation of two key organizations: the Viral Hemorrhagic Fever Consortium (vhfc.org) and the African Centre of Excellence for Genomics of Infectious Disease (acegid.org). ${ }^{16}$ The former brought about collaboration between the Lassa fever wards of both Kenema Government Hospital (Sierra Leone) and Irrua Specialist Training Hospital (Nigeria), whereas the latter, which is supported by the World Bank and H3Africa, is based at Redeemer's University in Nigeria. These organizations were instrumental in quelling the outbreak by utilizing polymerase chain reaction-based diagnostic tests to identify the first cases of Ebola in Sierra Leone in May 2014 and Nigeria in July 2014. ${ }^{16,17}$ The investment in infrastructure to support endemic public health challenges like Lassa virus has demonstrated the potential of domestic networks supported by international collaboration to respond to regional outbreaks that cannot be achieved in isolation, but by wellestablished collaborations.

\section{Microbiome research in H3Africa}

Microbiomes have been shown to play an important role in diseases. ${ }^{18-20}$ There are several microbiome projects within the H3Africa consortium, some of which are investigating the role of the microbiome in specific diseases, such as pneumonia in children or cervical cancer in women. As an example, the African Collaborative Center for Microbiome and Genomics Research (ACCME), based in Abuja, Nigeria, is a multicenter study of host germ line, somatic, and human papillomavirus (HPV) genomics and epigenomics, and the vaginal microenvironment and their association with cervical cancer. ${ }^{21}$ The center has enrolled over 12,000 women in prospective cohorts. One of ACCME's goals is to study the vaginal microbiota and its interaction with innate vaginal immunity in response to persistent high-risk (hr) HPV infection. To this end, they evaluated the association between hrHPV and the vaginal microbiome of HIV-negative and HIV-positive African women. Vaginal bacterial composition was characterized by deep sequencing of bar-coded $16 \mathrm{~S}$ rRNA gene fragments (V4) on Illumina MiSeq, and HPV was identified using the Roche Linear Array HPV genotyping test. The results of their linear discriminant analysis effect size algorithm suggested 
an association between prevalent hrHPV infection and a decreased abundance of Lactobacillus spp., with increased abundance of anaerobes particularly of the genera Prevotella and Leptotrichia in HIV-negative Nigerian women. ${ }^{22}$

Recently, they examined associations between prevalence and persistence of Mycoplasma spp. in the vaginal microbiota and prevalent as well as persistent hrHPV infections. For this study, they included HIV-negative Nigerian women who were tested for hrHPV infection using $\mathrm{SPF}_{25} / \mathrm{LiPA}_{10}$, and they identified $M$. genitalium and $M$. hominis in their vaginal microbiota, established by sequencing the V3-V4 hypervariable regions of the $16 \mathrm{~S}$ rRNA gene. They showed that persistent $M$. hominis in the vaginal microbiota was significantly associated with persistent hrHPV. ${ }^{23}$ Currently, ACCME studies are being conducted to characterize the microbial species composition of abundance in the vaginal microbiota of healthy women and to determine the epidemiological and behavioral predictors of types and stability of vaginal microbiota of African women and their association with persistent hrHPV infection. The results of these studies may contribute to advances in therapeutic intervention at the level of the vaginal microenvironment to "treat" or prevent persistent hrHPV infection, thus reducing the risk of progression to cervical intraepithelial neoplasia grade $2+$.

\section{Data generation and harmonization}

The H3Africa consortium is generating data of different types and formats. Almost all research projects are collecting clinically relevant and other phenotype data, as well as genomic data from the participants and in some cases from pathogens. The genomic data include whole-genome sequencing, exome sequencing, 16S rRNA sequencing (for microbiome studies), targeted sequencing, and data from genotyping or copy number variant arrays. The data constitute a valuable resource for disease studies in Africa and are being submitted to public repositories, such as the European Genome-Phenome Archive (EGA) and the European Nucleotide Archive (ENA). In order to ensure timely deposition of the data into the public domain and responsible sharing of the data, the H3Africa consortium developed a data-sharing, access, and release policy that is mindful of the challenges of large-scale genomic data analysis and interpretation in resource-limited settings and offers a generous timeline for data submission. According to the policy, after the genomic data have undergone quality control, researchers have 9 months to analyze the data before they have to be submitted to the public repositories. Thereafter, a further 12-month publication embargo is in place to protect the data generators. In order to implement these timelines, the H3Africa archive was established, hosted by $\mathrm{H} 3 \mathrm{ABioNet}$. The archive is a central repository of data in Africa, where researchers submit their data once they have been quality controlled. This provides the funders with a record of when the data are due to be submitted to the public repositories ( 9 months later). The archive team acts as the data-coordinating center, preparing the data for submission and liaising directly with the EGA or ENA.

In order to improve the usefulness of the data being generated and submitted by H3Africa researchers, it needs to be well curated and harmonized. The H3Africa consortium convened a phenotype harmonization working group that recommended 24 questions (phenotypic and demographic) be collected by all projects, which included 10 minimum required questions. However, even with these recommendations, a survey of case report forms across the consortium demonstrated that the questions in the core data categories were being asked in different ways or measurements taken or reported differently, meaning that the data outputs were not directly comparable. The working group, together with an ontology task force from $\mathrm{H} 3 \mathrm{ABioNet}$ and representatives from $\mathrm{H} 3$ Africa projects, used PhenX (https://www.phenxtoolkit.org) measures and their own expertise to develop standard measurements or questions and a set of guidelines for developing a case report form. A standard RedCap template implementing these standard questions has been developed for use by future studies. The ontology task force is also responsible for mapping clinical and genomic data to existing ontologies, such as phenotype, disease, experimental factor, and data-use ontologies prior to submission. This is to make the data more easily searchable and comparable. Metadata from projects and biospecimens will also be searchable in an H3Africa catalog to ensure the public can identify what data and biospecimens are available and how to request access to them. Access to all human data and biospecimens is controlled by the H3Africa Data and Biospecimen Access Committee.

In addition to attempts to harmonize data across all H3Africa projects, a subset of projects studying related phenotypes have self-organized to harmonize across their projects. A flagship example is the CVD working group discussed previously, which is retrospectively harmonizing data across six projects with research questions and data related to CVD risk. By using existing questions from each study, eg, related to smoking behavior, the data can be transformed into a single set of smoking-related variables. These can then be analyzed in over 50,000 African participants and will increase the power for discovery and replication genomewide association study analyses. 


\section{Reference population data and the H3Africa SNP array}

African data are poorly represented in public repositories. ${ }^{24}$ The majority of human genetic data in the public domain is derived from non-African populations, and most genotyping arrays were developed for populations of non-African descent. In order for precision medicine to become a reality in Africa, we need larger reference and control populations and more appropriate data generation tools. The H3Africa consortium realized the need for a better genotyping array that accounts for the larger genetic diversity and smaller haplotype blocks in African genomes, and set about designing an H3Africa array. In addition to African data from the 1000 Genomes Project ${ }^{25}$ and African Genome Variation Project, ${ }^{26}$ the consortium had 350 samples from diverse African populations sequenced at high coverage and additional samples with sequence data at medium coverage $(11 \times)$ were contributed by TrypanoGen, one of the H3Africa projects. High-coverage and low-coverage data were aligned and variants called were used for the array design. To reduce the final costs of the array, it includes $\sim 1.7$ million single nucleotide polymorphisms (SNPs) as base content from existing Illumina bead pools selected based on their applicability to studying African genomes (common SNPs in the African sequence data sets) and efficiency as tag SNPs. An additional 700,000 SNPs were selected from the sequence data to include novel but common African variants, SNPs of interest for specific diseases, and custom SNPs requested by H3Africa researchers. The array has recently been dispatched to the first H3Africa customers and will become commercially available.

Both the array and the associated reference panel for imputation provide much-needed tools for the analysis of genetic data from individuals of African descent. They also form the basis for genome-wide association studies for understanding the genetic basis of diseases in African populations and a reference data set for precision-medicine applications. The array design exercise also helped to develop skills and infrastructure on the continent for the analysis and interpretation of whole-genome sequence data. H3ABioNet has developed workflows for analysis and imputation of the array data, which can be deployed on a high-performance computing cluster or a cloud environment. Adoption of these workflows will ensure reproducibility and compatibility of data generated using the H3Africa array. Additionally, Illumina has committed equipment to an African laboratory to ensure the arrays can be run on the continent.

\section{Human and infrastructure capacity development}

The implementation of precision medicine requires substantial capacity for genomic research and translation into genomic medicine. One of the key outcomes of the H3Africa initiative has been in capacity development, both in human expertise and in equipment or research infrastructure. The consortium has a federated but coordinated training program, as each of the projects has its own training initiatives, while an H3Africa Education and Coordinated Training working group aims to harmonize and coordinate these activities. Over the first 5 years of the consortium's existence, more than 500 individuals were trained in many aspects of genomic research. These trainees are helping to create a pool of scientists capable of establishing large-cohort studies to improve our understanding of diseases in the African context. The consortium also has a fellows program for students and young academics, who regularly attend consortium meetings with special events arranged, such as career development workshops and symposia. Through the training program, the need to increase the level of skills in genomic medicine for health-care professionals was recognized. An important spin-off of some of the H3Africa training efforts has been the establishment of the African Genomic Medicine Training Initiative (https://training.h3abionet.org/AGMC 2016), which has successfully completed its first distance-based online training course in genomic medicine for nurses in Africa. A related course for clinicians is currently under development.

Through funding of individual research projects, recipients of H3Africa grants have had the opportunity to upgrade their research infrastructure significantly. This includes investments in training research and finance staff as well as in upgrading laboratories and equipment. Some of the projects have acquired equipment for high-throughput sequencing or genotyping, and many multisite projects have standardized their operating procedures for sample collection and DNA extraction. This has dramatically increased the ability of sites to process their own high-quality samples.

Another very important outcome of the H3Africa initiative is the development of biorepositories for housing of valuable samples. H3Africa funding has supported the upgrading of three regional biorepositories in western, eastern, and southern Africa to internationally accepted International Society for Biological and Environmental Repositories standards for the processing, archiving, and distribution of high-quality biological specimens for downstream genome analysis. These regional biorepositories have 
devised innovative and collaborative ways to overcome key challenges of establishing high-quality biorepositories that are captured in a special issue of Biopreservation and Biobanking ${ }^{27-30}$ These innovations include establishing reliable power through several backup strategies that combine the national power grid with solar panels, backup generators, and inverters, working with different regulatory and ethical legal bodies to ensure that processes are in place for responsible sharing of specimens that respects individual rights and national laws, engaging shipping companies in the process of affordable shipping, joint partnerships and responsibility in acquiring import permits and licenses, and adequate tracking of samples. These are all important resources for training and supporting clinical sites engaged in H3Africa projects in acquiring and shipping high-quality biological samples to the biorepositories for long-term storage and subsequent distribution to the network of scientists involved in genomic research.

\section{Working toward sustainability}

Many of the H3Africa consortium activities have been funded through the NIH Common Fund, which has a 10-year cycle. After this time, the consortium needs to source alternative funds or reduce its current level of activity in line with alternate funding sources. Therefore, the next 4 years will see a strong focus on sustainability. An H3Africa sustainability working group has been established and is engaging with governments and funding agencies to develop a strategy to ensure a long-term commitment to genomic research in Africa. Ideally, African governments should invest long term in improving and maintaining research infrastructure at local institutions, which can then attract research funding from local and international funding agencies. Building research and human capacity increases confidence and attractiveness for additional investment. The regional biorepositories are part of the sustainability effort, and have established infrastructures that will continue to be a resource in Africa, providing high-quality samples and services to extended research endeavors within the continent and globally as well as being a valuable resource for consultations on genomic studies and training.

\section{How is H3Africa contributing to the feasibility of precision medicine?}

Some of the key achievements of the H3Africa consortium have been highlighted herein. The consortium has developed an SNP array with enriched custom content of over 700,000 variants that were derived from whole-genome sequences of individuals of African descent. More than 30,000 samples have been committed for processing on the new array. One example is the SIREN project, which is performing genomewide association studies to probe the association between over a million variants and stroke phenotypes. With this, new genes associated with stroke, especially ischemic stroke and its subtypes, can be identified and will form a template for gene editing in future prevention or treatment of stroke. Other projects that are using the array include kidney disease, CafGen, TrypanoGen, AWI-Gen, and a sickle-cell disease project.

Though the data generated from H3Africa projects are paving the way toward increased knowledge of various diseases, these diseases are complex, with multiple variants and environmental factors playing a role. Therefore, direct application to improving health is not straightforward. In places where implementation of precision medicine is under way, eg, the USA, challenges have been cited, including limited evidence for clinical use and lack of data from diverse populations. ${ }^{31}$ This has highlighted the need for implementation science and development of guidelines to support decisionmaking in clinical settings. The more valuable contribution of the H3Africa consortium outputs to precision medicine is in the sets of phenotyped cohorts across different ethnic groups, background reference population genetic data, and the development of the skills and infrastructure to collect, store, harmonize, analyze, and interpret the data. As alluded to previously, in addition to improving our understanding of non-communicable diseases, the infrastructure developed by the H3Africa consortium has also equipped laboratories in Africa to respond more rapidly to pathogen outbreaks. The Ebola outbreak in west Africa in 2014 was a wake-up call for African scientists to prepare themselves with both equipment and skills to improve the response rate to curb outbreaks. Scientists involved in one of the H3Africa projects and the World Bank-funded ACEGID program based at Redeemer's University were instrumental in diagnosing and responding to Ebola cases in the region. ${ }^{32}$ The first diagnosis of Ebola in Nigeria and Sierra Leone was made by researchers at ACEGID-established laboratories, who were able to track the origin and evolution of the virus in west Africa. ${ }^{33}$

\section{Overcoming ethical challenges and community engagement}

Current ethics research in H3Africa focuses on understanding and improving methods for conducting genomic research in an ethical way. In Africa, ongoing research aims to understand various methods of improving the informed consent 
process through knowledge and awareness among research participants and community engagement. The H3Africa consortium has brought members of ethics boards from around the continent for regular meetings to address consistency and eduction in developing an appropriate and ethical consent framework. Ethics experts continue to discuss in detail how these can be applied in the African setting.

Although H3Africa focuses on generating populationlevel research results, implementation of precision medicine in the clinical setting faces some important ethical challenges that will need to be considered prior to its adoption. The most pertinent ethical challenge relates to questions about resource allocation and ensuring that the introduction of precision medicine in African clinics does not take funding away from other forms of health care that have greater potential to affect the health of a larger group of people positively. For instance, if competition over resources would result in favoring precision medicine, which may help a few, and take funding away from prenatal care, which could help many, then the introduction of precision medicine is not desirable or even ethical. Yet there are scenarios imaginable in which the introduction of precision medicine may be cost-effective, eg, in preventing toxic drug reactions because individuals are identified as "slow metabolizers". Not only could this influence national health policies, as it did in the case of Botswana, but such knowledge could directly affect the lives of individuals and prevent the expense of costly hospital admissions to deal with the side effects of drug toxicity.

One clear recommendation emanating from justice literature is that in order for research and care to contribute to reducing global health inequality effectively, interventions need to improve the fate of the worst off. ${ }^{34}$ In the context of the introduction of precision medicine initiatives to African clinics, the guide should be that there is real potential for such introduction to impact on the health of societies' poorest or most marginalized individuals and communities, or (perhaps more importantly) that such introduction does not negatively impact their health. More work needs to be done to understand this dynamic better, and to develop evidencebased cost-benefit assessments to explore whether and when the introduction of precision medicine is desirable and ethical.

The H3Africa SAX study noted that a community advisory board $(\mathrm{CAB})$ is one mechanism of engaging the community on such issues as precision medicine. While it is possible that $\mathrm{CABs}$ have important benefits, this may depend on whether they really are able to engage with communities. Investigators described the contributions made by the study $\mathrm{CAB}$, emphasizing four broad discussion topics that emerged during the $\mathrm{CAB}$ : informed consent procedures, recruitment strategies, patient illness beliefs and stigma experiences, and specific ethical concerns relating to the project. ${ }^{14}$ The $\mathrm{CAB}$ contributed to consent processes and materials and to recruitment strategies, advised on ways of minimizing stigma and discrimination, and helped the researchers to promote the respect and dignity of research participants and their community. ${ }^{14}$

\section{Conclusion and future prospects}

The H3Africa consortium consists of over 500 members, covering more than 30 of the 55 countries in Africa. The initiative has celebrated many successes and has exceeded the expectations of all those involved. While the key focus for individuals is seeing the objectives of the projects met, so much more has been achieved as a collective: the H3Africa consortium as a whole is truly greater than the sum of its parts. The collaborative nature of the consortium and its working groups have helped to solve what seemed like insurmountable challenges 5 years ago. They have worked together, bringing in diverse skills to develop policies for the fair and equitable sharing of data and biospecimens for the greater good of science, to develop guidelines for community engagement, an ethics and governance framework for best practice in genomic research and biobanking in Africa, and to build the next generation of scientists capable of high-quality genomic research.

The second round of H3Africa projects is now under way, and additional spin-off projects emanating from data generated in the first round have been initiated. For example, data sequenced at high coverage within the H3Africa consortium for the array design are being analyzed in a collaborative effort to improve our understanding of African genomes and novel variations. Research activity in the domain of pharmacogenomics is underrepresented in African populations, yet it provides an opportunity for the discovery of new drug targets and functionally important alleles in known ADME (absorption, distribution, metabolism, and excretion) genes. The whole-genome sequence data generated by the H3Africa consortium will be used for novel variant mining in ADME genes, and in conjunction with SNP array data and copy number variation analysis, to assess the frequencies of functionally important variants across different African populations. This knowledge will be applied to decision-making processes to inform essential drug lists in different countries and influence public precision medicine decisions on drugs most likely to have maximum benefit in promoting the health of a population. 


\section{Disclosure}

The authors report no conflicts of interest in this work.

\section{References}

1. Biankin AV. The road to precision oncology. Nat Genet. 2017; 49(3):320-321.

2. Gómez-López G, Dopazo J, Cigudosa JC, Valencia A, Al-Shahrour F. Precision medicine needs pioneering clinical bioinformaticians. Brief Bioinform. Epub 2017 Oct 25.

3. Petrovski S, Goldstein DB. Unequal representation of genetic variation across ancestry groups creates healthcare inequality in the application of precision medicine. Genome Biol. 2016;17(1):157.

4. Rotimi C, Abayomi A, Abimiku A, et al. Enabling the genomic revolution in Africa. Science. 2014;344(6190):1346-1348.

5. Mulder NJ, Adebiyi E, Alami R, et al. H3ABioNet, a sustainable panAfrican bioinformatics network for human heredity and health in Africa. Genome Res. 2015;26(2):271-277.

6. McKee M, Haines A, Ebrahim S, et al. Towards a comprehensive global approach to prevention and control of NCDs. Global Health. 2014;10:74.

7. Owolabi MO, Mensah GA, Kimmel PL, et al. Understanding the rise in cardiovascular diseases in Africa: harmonising H3Africa genomic epidemiological teams and tools. Cardiovasc J Afr. 2014;25(3): 134-136.

8. Peprah E, Wiley K, Troyer J, et al. Building a platform to enable NCD research to address population health in Africa: CVD working group discussion at the Sixth H3Africa Consortium Meeting in Zambia. Glob Heart. 2016;11(1):165-170.

9. Ramsay M, Crowther N, Tambo E, et al. H3Africa AWI-Gen collaborative centre: a resource to study the interplay between genomic and environmental risk factors for cardiometabolic diseases in four sub-Saharan African countries. Glob Health Epidemiol Genom. 2016;1:e20.

10. Gómez-Olivé FX, Ali SA, Made F, et al. Stark regional and sex differences in the prevalence and awareness of hypertension: an H3Africa AWI-Gen study across 6 sites in sub-Saharan Africa. Glob Heart. 2017;12(2): 81-90.

11. Akinyemi R, Arnett DK, Tiwari HK, et al. Interleukin-6 (IL-6) rs1800796 and cyclin dependent kinase inhibitor (CDKN2A/CDKN2B) rs2383207 are associated with ischemic stroke in indigenous west African men. J Neurol Sci. 2017;379:229-235.

12. Owolabi M, Salaam K, Ogunniyi A, et al. SIREN: Aim on Clear Canvas Enriched Stroke phenotyping (ACCESS) [software]. Nigeria patent NG/ $\mathrm{PT} / \mathrm{NC} / 2016 / 2007.2016$.

13. Campbell MM, Sibeko G, Mall S, et al. The content of delusions in a sample of South African Xhosa people with schizophrenia. BMC Psychiatry. 2017;17:41.

14. Campbell MM, Susser E, de Vries J, et al. Exploring researchers' experiences of working with a researcher-driven, population-specific community advisory board in a South African schizophrenia genomics study. BMC Ethics. 2015;16:45.
15. World Health Organization. Ebola Situation Reports. Available from: http://www.who.int/csr/disease/ebola/situation-reports/archive/en/. Accessed November 28, 2017.

16. Yozwiak NL, Happi CT, Grant DS, et al. Roots, not parachutes: research collaborations combat outbreaks. Cell. 2016;166(1):5-8.

17. Schieffelin JS, Shaffer JG, Goba A, et al. Clinical illness and outcomes in patients with Ebola in Sierra Leone. N Engl J Med. 2014; 371(22):2092-2100.

18. Selber-Hnatiw S, Rukundo B, Ahmadi M, et al. Human gut microbiota: toward an ecology of disease. Front Microbiol. 2017;8:1265.

19. Hall AB, Tolonen AC, Xavier RJ. Human genetic variation and the gut microbiome in disease. Nat Rev Genet. 2017;18(11):690-699.

20. Arrieta MC, Stiemsma LT, Amenyogbe N, Brown EM, Finlay B. The intestinal microbiome in early life: health and disease. Front Immunol. 2014;5:427.

21. Adebamowo SN, Dareng EO, Famooto AO, et al. Cohort profile: African Collaborative Center for Microbiome and Genomics Research's (ACCME) human papillomavirus (HPV) and cervical cancer study. Int J Epidemiol. 2017;46(6):1745.

22. Dareng EO, Ma B, Famooto AO, et al. Prevalent high-risk HPV infection and vaginal microbiota in Nigerian women. Epidemiol Infect. 2016;144(1):123-137.

23. Adebamowo SN, Ma B, Zella D, et al. Mycoplasma hominis and Mycoplasma genitalium in the vaginal microbiota and persistent high-risk human papillomavirus infection. Front Public Health. 2017;5:140.

24. Popejoy AB, Fullerton SM. Genomics is failing on diversity. Nature. 2016;538(7624):161-164

25. Auton A, Brooks LD, Durbin RM, et al. A global reference for human genetic variation. Nature. 2015;526(7571):68-74.

26. Gurdasani D, Carstensen T, Tekola-Ayele F, et al. The African Genome Variation Project shapes medical genetics in Africa. Nature. 2015;517(7534):327-332.

27. Abimiku A, Mayne E, Jaloba M, et al. H3Africa biorepository program: supporting genomics research on African populations by sharing highquality biospecimens. Biopreserv Biobank. 2017;15(2):99-102.

28. Croxton T, Swanepoel C, Musinguzi H, et al. Lessons learned from biospecimen shipping among the Human Heredity and Health in Africa (H3Africa) biorepositories. Biopreserv Biobank. 2017;15(2):104-111.

29. Kyobe S, Musinguzi $\mathrm{H}$, Lwanga $\mathrm{N}$ et al. Selecting a laboratory information management system for biorepositories in low- and middle-income countries: the H3Africa experience and lessons learned. Biopreserv Biobank. 2017;15(2):111-115.

30. Beiswanger C, Abimiku A, Carstens N, et al. Accessing biospecimens from the H3Africa consortium. Biopreserv Biobank 2017;15(2):95-98.

31. Korngiebel DM, Thummel KE, Burke W. Implementing precision medicine: the ethical challenges. Trends Pharmacol Sci. 2017;38(1):8-14.

32. Folarin OA, Happi AN, Happi CT. Empowering African genomics for infectious disease control. Genome Biol. 2014;15(11):515.

33. Gire SK, Goba A, Andersen KG, et al. Genomic surveillance elucidates Ebola virus origin and transmission during the 2014 outbreak. Science. 2014;345(6202):1369-1372.

34. Pratt B, Hyder A. Governance of transnational global health research consortia and health equity. Am J Bioeth. 2016;16(10):29-45.
Pharmacogenomics and Personalized Medicine

\section{Publish your work in this journal}

Pharmacogenomics and Personalized Medicine is an international, peerreviewed, open access journal characterizing the influence of genotype on pharmacology leading to the development of personalized treatment programs and individualized drug selection for improved safety, efficacy and sustainability. This journal is indexed on the American Chemical
Society's Chemical Abstracts Service (CAS). The manuscript management system is completely online and includes a very quick and fair peer-review system, which is all easy to use. Visit http://www.dovepress. com/testimonials.php to read real quotes from published authors. 